INPLASY

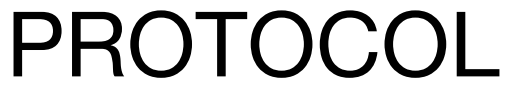

To cite: Wang et al.

Comparative efficacy of bariatric surgery for type 2 diabetes mellitus: A protocol for systematic review and network meta-analysis. Inplasy protocol 202050053. doi: 10.37766/inplasy2020.5.0053

Received: 13 May 2020

Published: 13 May 2020

Corresponding author:

Xixiong Wang

xixiongwang@126.com

Author Affiliation:

Boao Evergrande International Hospital, Qionghai, 571437, China.

Support: Hainan Health Science and Education Project (No. 19A200043)

Review Stage at time of this submission: Formal screening of search results against eligibility criteria.

Conflicts of interest:

None.

\section{Comparative efficacy of bariatric surgery for type 2 diabetes mellitus: A protocol for systematic review and network meta-analysis}

Wang, X1; Chen, C²; Zheng, B3; Yang, X4; Zhang, X5; Yang, C6.

Review question / Objective: To evaluate the effectiveness of bariatric surgery for patients with type 2 diabetes mellitus. Condition being studied: The effectiveness of bariatric surgery for patients with type 2 diabetes mellitus has been confirmed. Different surgical procedures are available, although the comparative effectiveness of these approaches for the treatment of type 2 diabetes mellitus is still unknown.

Information sources: We systematically searched for eligible randomized controlled trials in PubMed, Embase, and The Cochrane Library without search date and language restrictions.

INPLASY registration number: This protocol was registered with the International Platform of Registered Systematic Review and Meta-Analysis Protocols (INPLASY) on 13 May 2020 and was last updated on 30 September 2020 (registration number INPLASY202050053).

\section{INTRODUCTION}

Review question / Objective: To evaluate the effectiveness of bariatric surgery for patients with type 2 diabetes mellitus.
Condition being studied: The effectiveness of bariatric surgery for patients with type 2 diabetes mellitus has been confirmed. Different surgical procedures are available, although the comparative effectiveness of 
these approaches for the treatment of type 2 diabetes mellitus is still unknown.

\section{METHODS}

Participant or population: Patients with type 2 diabetes.

Intervention: Different bariatric surgical procedures.

\section{Comparator: Medical treatment.}

Study designs to be included: Published randomized controlled trials.

Eligibility criteria: Randomized controlled trails (RCTs) including adults with COPD receiving bariatric surgery in any type of procedures compared with each other or medical treatment will be eligible for inclusion. Only studies reported in English will be included.

Information sources: We systematically searched for eligible randomized controlled trials in PubMed, Embase, and The Cochrane Library without search date and language restrictions.

Main outcome(s): Type 2 diabetes mellitus (T2DM) remission analyzed at 12 months and 60 months.

Additional outcome(s): BMI, HbA1c (\%), and percentage excess weight loss $(\%$ EWL); All the outcomes will be analyzed at 12 months and 60 months.

Quality assessment / Risk of bias analysis: The risk of bias will be assessed using the Cochrane Collaboration's tool for randomized controlled trials.

Strategy of data synthesis: Fixed and random effects models will be used to pool the data, and $R$ 3.5.0 software and Stata version 14.0 (College Station, TX) will be used to conduct the data synthesis.

Subgroup analysis: None planned.

Sensibility analysis: Robustness of primary findings will be tested with sensitivity analysis by excluding trials with high risk of bias, by performing leave-one-out analysis, and by excluding studies requiring data imputation.

Language: English.

Country(ies) involved: China.

Other relevant information: 1. Xixiong Wang, MD, Department of surgical oncology, Boao Evergrande International Hospital, Qionghai, 571437, China. E-mail address: xixiongwang@126.com 2. Cunren Chen, MD, Department of endocrinology, Hainan General Hospital, Haikou, China. Email address: page20062006@163.com 3. Buping Zheng, MD, Department of surgical oncology, Boao Evergrande International Hospital, Qionghai, 571437, China. E-mail address: zhengbuping@163.com 4. Xiaolong Yang, MD, Department of surgical oncology, Boao Evergrande International Hospital, Qionghai, 571437, China. E-mail address: 25700147@qq.com 5. Xiaoxin Zhang, Department of surgical oncology, Boao Evergrande International Hospital, Qionghai, 571437, China. E-mail address: zhangxiaostar@126.com 6. Chenchen Yang, MD, Department of surgical oncology, Boao Evergrande International Hospital, Qionghai, 571437, China. E-mail address: 352394234@qq.com .

Keywords: Type 2 diabetes mellitus.

Contributions of each author:

Author 1 - Xixiong Wang.

Author 2 - Cunren Chen

Author 3 - Buping Zheng.

Author 4 - Xiaolong Yang.

Author 5 - Xiaoxin Zhang.

Author 6 - Chenchen Yang. 